\title{
La vivienda de interés social y el mercado inmobiliario: agentes y dinámicas del programa Minha Casa Minha Vida en la ciudad de Pelotas (2009-2016)
}

\author{
Social housing and the real estate market: agents and \\ dynamics of the Minha casa Minha vida program in \\ Pelotas (Brazil)
}

Natalia Daniela Soares Sá Britto² [1] y Oscar Alfredo Sobarzo Miño³ (i)

\begin{abstract}
RESUMEN
En este artículo se analiza la producción de viviendas en la ciudad brasileña de Pelotas, en el marco del Programa Minha Casa Minha Vida (PMCMV), entre 2009 y 2016. El análisis se inicia situando la creación del programa en el contexto político-económico de la primera década del siglo XXI, como una retomada del papel regulador y subsidiario del Estado en el proceso de acumulación de capital. Son abordadas las características generales del PMCMV y analizado su impacto en la ciudad según las principales modalidades de producción de viviendas para diferentes grupos de ingresos. En el texto se constata que el actuar de diferentes agentes, asociados cuando les resultaba conveniente, y las dinámicas de producción de viviendas, significaron la ampliación del área urbanizada, con importante expansión periférica, y la valorización del suelo y de las propias empresas en función de la financiarización del proceso, sin provocar la disminución real del déficit habitacional.

Palabras claves: Vivienda de interés social, Programa Minha Casa Minha Vida, Mercado inmobiliario, Valorización del suelo, Expansión periférica
\end{abstract}

\begin{abstract}
In this article, housing production in the Brazilian city of Pelotas is analyzed, within the Minha Casa Minha Vida Program (MCMV), between 2009 and 2016. The analyses begin by placing the program's creation in the political-economic context of the first decade of the 21st century, characterized by a resurgence of the state's regulatory and auxiliary role in the process of capital accumulation. The general characteristics of the MCMV are addressed and their impact on the city is analyzed according to the main housing production modalities for different income groups. The article shows that the action of different agents, establishing partnerships when necessary, and the dynamics of housing production, generated an expansion of the urbanized area, with significant peripheral expansion, and the increase in land values and in the value of firms themselves, due to the financialization, without, however, addressing the housing deficit.
\end{abstract}

Keywords: Social housing, Minha Casa Minha Vida Program, Real estate Market, Land values, Peripheral expansion

Financiado por Coordenação de Aperfeiçoamento de Pessoal de Nível Superior - Brasil (CAPES) mediante la concesión de una Beca de Doctorado y por Fundação de Amparo à Pesquisa do Estado de S. Paulo (FAPESP) a través de la investigación postdoctoral vinculada al proyecto temático "Fragmentación socioespacial y urbanización brasileña: escalas, vectores, ritmos, formas y contenidos" (FragUrb) Processo nº 2019/05225-7 
En este artículo se abordan las características de la producción de viviendas en la ciudad de Pelotas, en el contexto del Programa Minha Casa Minha Vida (PMCMV) entre 2009 y 2016. En primer lugar se presenta una contextualización de la situación brasileña en la primera década del siglo actual que lleva a la creación del programa público de estímulo a la construcción de viviendas. En el contexto político-económico que pasaba Brasil, el impulso a la retomada del papel del Estado en la regulación e inducción del proceso de acumulación de capital favoreció a los sectores de la construcción civil y al sector patrimonial-rentista, obedeciendo al pacto de poder que sustentaba al gobierno.

Las características institucionales del PMCMV y sus modalidades permiten visualizar su importancia en el proceso de producción de viviendas y del espacio urbano como un todo. En ese sentido, el objetivo del artículo es destacar la acción de diferentes agentes (privados y públicos) que actúan con variados objetivos y estrategias, ocasionando una dinámica de transformaciones y permanencias en la producción y valorización de las empresas y del suelo urbano.

En términos generales, el análisis presentado en el artículo demuestra que el impacto del PMCMV en Pelotas y las acciones y estrategias de los diferentes agentes se tradujeron en un elevado volumen de unidades habitacionales construidas, que llevaron a la ampliación del área urbanizada, con importante expansión periférica, y provocaron la valorización del suelo y de las propias empresas en función de la financiarización del proceso, sin provocar la disminución real del déficit habitacional existente en la ciudad.

\section{Metodología}

Para identificar y analizar la actuación de los agentes se utilizó una variedad de fuentes de información, instrumentos de recopilación y procedimientos técnicos, tanto cualitativos como cuantitativos, que son fundamentales para operar un enfoque analítico sobre las características formales y funcionales de los procesos, agentes y acciones destacados en nuestro estudio.

Entre estos procedimientos, destacamos: a) Recopilación de información y observaciones in loco, basadas en el uso de diarios de campo, donde se realizaron descripciones periódicas de las principales mesorregiones de planificación de la ciudad de Pelotas entre los años 2012 y 2016. Estas descripciones hicieron posible monitorear los cambios en la morfología urbana; b) Recopilación de información sobre tipologías, materiales y características de los sistemas de construcción a través de visitas a los sitios web de empresas constructoras y desarrolladores, así como a través del acceso a las memorias descriptivas de los proyectos; c) Análisis y tabulación de datos estadísticos primarios y secundarios, analizados a través de variables continuas y en series temporales y transversales. Entre ellos, destacamos la sistematización de la emisión de guías del Impuesto de Transmisión de Bienes Raíces (ITBI), utilizado como fuente para el análisis e identificación de los vectores de valorización del suelo urbano e inmobiliaria, así como los datos sobre la distribución de recursos y unidades contratadas por el PMCMV en Pelotas, cuya fuente fue el Ministerio de las Ciudades; d) Análisis de registros y certificados notariales y la situación fiscal y catastral de empresas y los lineamientos del PMCMV, el III Plan Director de Pelotas y la ley orgánica municipal, como estrategia para identificar capitales, agentes y sus relaciones con el plan de regulación urbana. 


\section{Políticas sectoriales y producción del espacio urbano en Brasil}

Al iniciar el siglo XXI, Brasil pasó por un cambio político y por una reformulación de su política económica. El papel del Estado fue gradualmente retomado como un instrumento clave para garantizar el crecimiento económico, estimulando el ahorro interno, tasas de interés moderadas y un tipo de cambio competitivo, al mismo tiempo en que se implementó un conjunto de políticas económicas, sociales y sectoriales orientadas a la ampliación del mercado de trabajo, de la capacidad de crédito y del consumo interno.

Esa política, establecida en Brasil especialmente a partir de 2005 pasó a ser denominada como neodesarrollismo, caracterizando, según Bresser-Pereira (2010), una actualización de las teorías de desarrollo estructuralistas del siglo pasado en articulación con la teoría keynesiana, conformando una estrategia alternativa de desarrollo frente a la ortodoxia convencional. Entretanto esa interpretación merece reparos, ya que las medidas adoptadas no significaron una ruptura con la lógica neoliberal anterior, configurando un modelo híbrido al articular liberalización financiera y monetaria, con políticas sectoriales de crecimiento a corto plazo (Gonçalves, 2012). Según Pinassi (2013) se trató de una manera de administrar los daños causados por el neoliberalismo mediante la recomposición de la función pública de "alivio social", especialmente a partir de la generación de empleo, de la valorización del sueldo mínimo y de redistribución de la renta, pero sin abandonar una política monetaria centrada en el control de la inflación.

De acuerdo con Bastos (2012), las estrategias de desarrollo después de la crisis del neoliberalismo en Brasil llevaron a la formulación de por lo menos dos alternativas que rescataban el papel del Estado: el desarrollismo exportador o nuevo desarrollismo, introducido en el primer mandato de Luiz Inácio Lula da Silva (2003-2006), fundamentado en el sector privado y con gran correspondencia con las medidas del gobierno anterior; $y$ el desarrollismo distributivo o "social-desarrollismo" orientado por el Estado y caracterizado por la importancia de la distribución del ingreso y de las políticas sociales de reducción de la pobreza para la ampliación del mercado de consumo interno, medidas características del segundo mandato de Lula (2007-2010).

En ese sentido fueron implementadas políticas públicas de transferencia de renta, de aumento del sueldo mínimo y de expansión del crédito, que significaron la ampliación del poder de compra de una parte significativa de los brasileños de menor poder adquisitivo, estimulando el mercado interno (Araújo, 2013). De igual forma, la concesión de beneficios fiscales, la creación de líneas de crédito y financiamientos de largo plazo para empresas en el Banco Nacional de Desenvolvimento Económico (BNDES) y las inversiones públicas en obras de infraestructura social, urbana, logística y energética, constituyeron medidas estratégicas para incentivar la producción industrial. Pese a ello, la política monetaria y el peso del comercio internacional ratificaron y fortalecieron a Brasil como de productor y exportador de bienes primarios en una economía globalizada.

En este contexto fue anunciado en 2007 el Programa de Aceleración del Crecimiento (PAC), como un conjunto de políticas económicas y de planificación estratégica orientadas al crecimiento económico mediante la retomada de las inversiones públicas y privadas en sectores estructurales, estimulando la construcción civil como estrategia de generación de empleos y renta. En su conjunto esas medidas repercutieron en la estabilización financiera del país ya que significaron 
un mayor compromiso del Estado con reformas en el ambiente de negocios y licitaciones, así como una participación presupuestaria que inyectó vultuosos recursos públicos y abrió nuevos campos para la reproducción del capital, tanto en la modernización de la base técnica-científica e informacional del territorio, como en la expansión de la construcción civil.

En marzo de 2009, dos años después del lanzamiento del PAC, se lanza el Programa Minha Casa Minha Vida (PMCMV) basado en una amplia articulación entre el Estado y las principales empresas constructoras del país para asignar recursos, créditos y subsidios para la producción y compra de viviendas sociales para familias con ingresos de hasta 10 sueldos mínimos. Al año siguiente, el PMCMV se integró en la segunda etapa del Programa de Aceleración para el Crecimiento (PAC-2)4, lo que permitió la expansión de los recursos para para financiar viviendas sociales.

Es importante señalar que el anuncio del PAC-2 y especialmente del PMCMV fue realizado en un contexto global específico generado por la crisis subprime en Estados Unidos, por lo cual tuvo como propósito generar una reacción momentánea a las dificultades coyunturales mediante la inducción del crecimiento económico interno, ampliando el volumen de crédito y los recursos públicos inyectados en el programa. Si el PAC-1 consideró un presupuesto de $\mathrm{R} \$ 657.000$ millones, el PAC-2 previó R\$950.000 millones hasta 2014.

El PMCMV surge como una oportunidad para conjugar por lo menos dos necesidades. Por un lado ayudaba a enfrentar las necesidades de vivienda social y por otro auxiliaba a las empresas de construcción frente al escenario de crisis. Según Cardoso y Aragão (2014) el sector de la construcción civil fue elegido para estimular la economía por sus efectos multiplicadores y por la amenaza que enfrentaban las empresas del sector en la bolsa de valores. De este modo la combinación de una justificativa social y los requerimientos del sector productivo llevó al gobierno a actuar directamente en estímulo del crecimiento económico, del consumo interno y de los indicadores de empleo especialmente después de 2009. Así fue promovido un nuevo ciclo de producción mediante la construcción de infraestructura urbana, logística y energética en lugares estratégicos, y por medio de programas habitacionales y de urbanización que articulaban políticas sociales (con expansión del ingreso y de la demanda) con las necesidades del mercado (financiamiento y crédito de largo plazo).

\section{Formato institucional, modalidades y etapas del Programa Minha Casa Minha Vida}

El Programa Minha Casa Minha Vida (PMCMV), oficializado en 2009, representa una coalición entre intereses gubernamentales, sectoriales y financieros articulados por el sistema nacional de financiamiento habitacional. A través de operaciones apoyadas en aportes financieros provenientes de fondos públicos, el PMCMV moviliza los ahorros familiares, sobretodo de los grupos de ingresos bajos y medios, para el financiamiento de viviendas propias por el mercado, utilizan-

Entre las nuevas medidas definidas en el PAC-2 fueron contemplados recursos para la expansión y recuperación de carreteras, ferrovías, hidrovías, puertos y aeropuertos; así como para la construcción de hidroeléctricas y termoeléctricas, explotación de petróleo y gas natural y revitalización de la industria naval. 
do subsidios para garantizar la ampliación de la demanda. Además este programa habitacional estuvo apoyado en la rebaja de las tasas de interés y exenciones fiscales en las operaciones de producción y comercialización de los inmuebles.

Del punto de vista operativo, el programa se organiza en dos subprogramas nacionales: Programa Nacional de Habitación Urbana (PNHU) y Programa Nacional de Habitación Rural (PNHR). El PNHU se destina a las familias con ingresos mensuales de hasta 10 sueldos mínimos ${ }^{5}$ residentes en áreas urbanas, con la siguiente estratificación: i) familias con ingresos hasta 3 salarios mínimos - clasificadas en el grupo 1 del programa - beneficiarias de subsidios de hasta $90 \%$ del valor de la vivienda provenientes de diferentes fondos públicos; ii) familias con ingresos entre 3 y 6 sueldos mínimos - clasificadas en el grupo 2 - beneficiadas con un subsidio de hasta $R \$ 29.000,00$ y condiciones especiales de financiamiento inmobiliario a partir de recursos del Fondo de Garantía por Tiempo de Servicio (FGTS 6 ); iii) familias con ingresos entre 6 y 10 sueldos mínimos - clasificadas en el grupo 3 - sin subsidios, pero beneficiadas con condiciones especiales de financiamiento. Por su parte, el PNHR se orienta a los residentes en áreas rurales mediante la concesión de subsidios para la construcción o reforma de sus viviendas; en esta modalidad los beneficiarios deben estar organizados en grupos y reciben recursos provenientes el presupuesto general de la nación.

En la perspectiva institucional, el Ministerio de las Ciudades (incorporado en 2019 al Ministerio del Desarrollo Regional) desempeño el papel de gestor y articulador del programa en conjunto con la Caixa Económica Federal (CEF), que es el agente financiero operador de los recursos destinados a la construcción de los proyectos y su fiscalización. Las empresas constructoras son las responsables técnicas por la realización completa de la operación: terreno, proyecto, permisos institucionales, construcción y comercialización. Las municipalidades actúan como agentes facilitadores realizando el catastro de la demanda para los proyectos del grupo 1, pudiendo incluso actuar en conjunto con las empresas disponiendo de terrenos o realizando llamadas públicas para proyectos (Brasil, 2014).

En síntesis, el PMCMV a partir de sus subprogramas, líneas de financiamientos y modalidades adquirió una escala considerable en Brasil, organizando un intrincado proceso de especialización y división de la producción inmobiliaria de la llamada "habitación social de mercado" (Shimbo, 2010).

\section{El PMCMV en la ciudad de Pelotas (2009-2016)}

La ciudad de Pelotas se localiza en el estado brasileño de Rio Grande do Sul, aproximadamente a $250 \mathrm{~km}$ al sur de la capital Porto Alegre. Según el último censo disponible, en 2010 la ciudad contaba con una población de 306.193 habitantes $^{7}$. El municipio de Pelotas se destaca como el

Es importante destacar que, durante sus 15 años de existencia, el PMCMV ha pasado por tres fases distintas. En cada una de ellas hubo cambios con respecto a los intervalos de ingresos de los beneficiarios, así como en las condiciones de financiamiento. Según las reglas actuales, la indexación ya no se realiza con el salario mínimo nacional y las familias con un ingreso mensual de hasta R 9 mil pueden participar en el programa, además de crear una nueva modalidad para familias con un ingreso de hasta $\mathrm{R} \$ 2,350.00$ (grupo 1,5).

EI FGTS es un fondo creado en 1966 que se destina a los trabajadores despedidos sin justa causa. Parte de los recursos de ese fondo se destina para las políticas habitacionales.

Según las proyecciones oficiales, en 2018 el municipio de Pelotas contaba con una población total (urbana y rural) de 341.648 habitantes. 
tercero en tamaño poblacional de Rio Grande do Sul, siendo precedido apenas por la capital y por Caxias do Sul.

Según datos de la Caixa Económica Federal, banco público que actúa como agente inmobiliario, la demanda por viviendas en el municipio alcanzaba 10.572 unidades en 2009 , constituyendo el segundo municipio con mayor carencia habitacional en el estado, representando $5 \%$ de la demanda total, especialmente concentrada en los estratos de ingresos de hasta tres sueldos mínimos. En el mismo sentido, según el Plan Local de Vivienda de Interés Social elaborado por la municipalidad de Pelotas en 2012, aproximadamente $90 \%$ de los 12.694 inscritos para acceder a una vivienda correspondían a familias con ingresos de hasta un sueldo mínimo, y de estos $93 \%$ habitaban en domicilios con condiciones precarias, demostrando el elevado déficit habitacional de la ciudad ${ }^{8}$.

Desde 2001, con la creación del Programa de Arrendamiento Residencial (PAR), el sector inmobiliario de Pelotas mostraba un gran crecimiento y dinamismo en las formas de producción adoptadas, convirtiéndose en referencia regional en la producción de viviendas en función de la especialización, innovación tecnológica y know-how de las empresas locales (Soares, 2002; Chiarelli, 2014). Por otra parte, con el PMCMV, desde 2009, los agentes inmobiliarios locales ampliaron considerablemente su escala de actuación consolidando el sector en función de la acumulación propiciada por el gran volumen de capital movilizado que hasta 2016, según cifras del extinto Ministerio de las Ciudades, alcanzó $\mathrm{R} \$ 735.000 .000$ y significó la contratación de 11.435 unidades habitacionales.

En términos regionales ese volumen de construcción colocó a Pelotas en el quinto lugar entre los municipios con mayor número de viviendas producidas en la modalidad Empresas ${ }^{9}$ en Rio Grande do Sul, en tercer lugar en construcción para el grupo 2 de ingresos y sexto entre los municipios en la construcción de viviendas para el grupo 3 de ingresos. Además de esta expansión del mercado local se destacó la fuerte actuación de las empresas de Pelotas en la escala regional, siendo el municipio con mayor número de empresas actuantes en la construcción de viviendas para el grupo 1 de ingresos y con el mayor número de lanzamientos (25 emprendimientos).

En síntesis, las empresas de Pelotas pasaron por un proceso de especialización en la producción de viviendas populares y de ampliación de su capacidad productiva en escala local y regional, en función del volumen de recursos destinados por la política pública que representó el PMCMV.

\section{PMCMV Modalidad Empresas (Grupo 1)}

En el grupo 1 del PMCMV, destinado a viviendas para familias con ingresos de hasta 3 sueldos mínimos fueron contratadas 3.332 unidades habitacionales en Pelotas entre 2009 y 2016, distribuidas entre las modalidades Empresas, Entidades y Rural ${ }^{10}$. En la modalidad Empresas fue contratada a construcción de 2.800 viviendas, distribuidas en 10 emprendimientos, con una inversión total de $\mathrm{R} \$ 146.325 .641$ (94\% de la inversión destinada al grupo 1 de ingresos).

Trento y Leitão (2013) destacan que los datos sobre déficit habitacional en Pelotas son discrepantes según las diferentes fuentes utilizadas, calculándose que podría llegar a 33.000 viviendas considerando los domicilios precarios.

Modalidad del PMCMV destinada a familias con ingresos de hasta 3 sueldos mínimos con subsidio de hasta $90 \%$ del valor de la vivienda.

La modalidad Entidades se destina a familias con ingresos de hasta 3 sueldos mínimos organizadas en cooperativas, asociaciones u otros grupos sin fines de lucro, con subsidio de hasta $90 \%$ del valor de la vivienda. La modalidad Rural fue creada para atender las necesidades de la población de áreas rurales. 
De las cinco constructoras de capital local que operan en Pelotas en esta modalidad, tres ya actuaban antes de 2009 produciendo conjuntos residenciales ligados al Programa de Arrendamiento Residencial (PAR), demostrando una progresiva especialización del sector local en la producción de viviendas populares (Soares, 2002; Chiarelli, 2014). Esas tres empresas corresponden a Labore Ingeniería Ltda., Roberto Ferreira Comercial y Constructora y Serial Ingeniería y Estructuras.

Labore Ingeniería Ltda. es la empresa con mayor número de emprendimientos y de unidades contratadas por el PMCMV grupo 1 en Pelotas (2009-2016). Actuando en el mercado inmobiliario local desde la década de 1990, con el nombre Constructora Theo Bonow, la empresa se especializó en la incorporación y construcción residencial de alto estándar, siendo la responsable por uno de los primeros condominios horizontales en 1995, además de la promoción de departamentos residenciales en el centro de la ciudad.

Desde los años 2000 la empresa pasó a operar con su nombre actual, expandiendo su producción con el PAR, construyendo cinco emprendimientos con un total de 818 viviendas entre 2002 y 2008. Para 2015 la empresa había sido responsable por más de 7.000 unidades habitacionales en el estado de Rio Grande do Sul, siendo residencias populares o de alto estándar. Esa dinámica demuestra la diversificación de la empresa asociada a los programas públicos de financiamiento (PAR y PMCMV) y la ampliación productiva y geográfica de su mercado con la incorporación de otras ciudades del estado.

En la modalidad Empresas, Labore Ingeniería fue responsable por la producción de tres conjuntos residenciales en Pelotas con 760 viviendas y un presupuesto de $\mathrm{R} \$ 39.410 .061$. El primer emprendimento lanzado fue el Residencial Montevideo (Figura № 1), localizado la zona periférica norte de la ciudad, con 240 departamentos distribuidos en 12 bloques de cinco pisos. En 2013 la empresa concluyó las obras del Residencial Buenos Aires (Figura № 2), también de 240 unidades y con las mismas características estructurales, localizado en un área adyacente al Residencial Montevideo, aprovechando una reserva de suelo urbano de la empresa. Ese mismo año es iniciada la construcción del Residencial Roraima con 280 unidades distribuidas en 20 bloques de cinco pisos.

Figura $\mathrm{N}^{\circ} 1$

Residencial Montevideo, Labore Ingeniería Ltda.

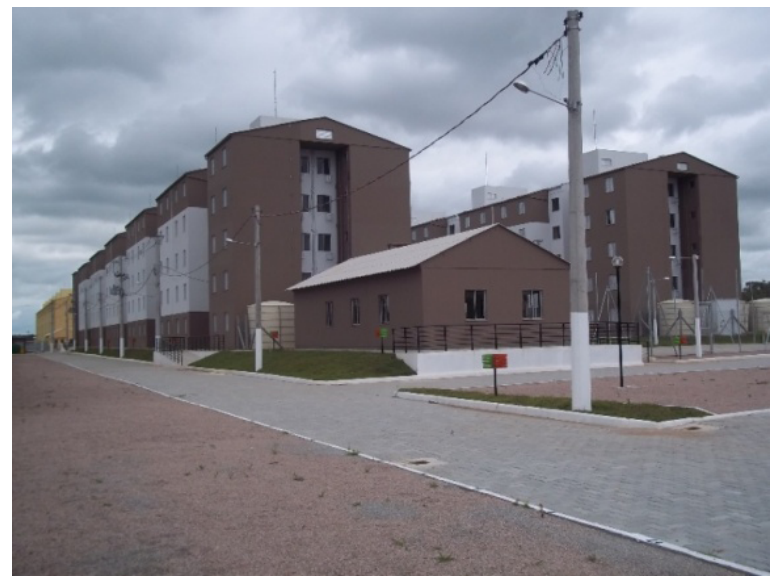

Fuente: Labore Ingeniería (2013). 
Figura № 2

Residencial Buenos Aires, Labore Ingeniería Ltda.

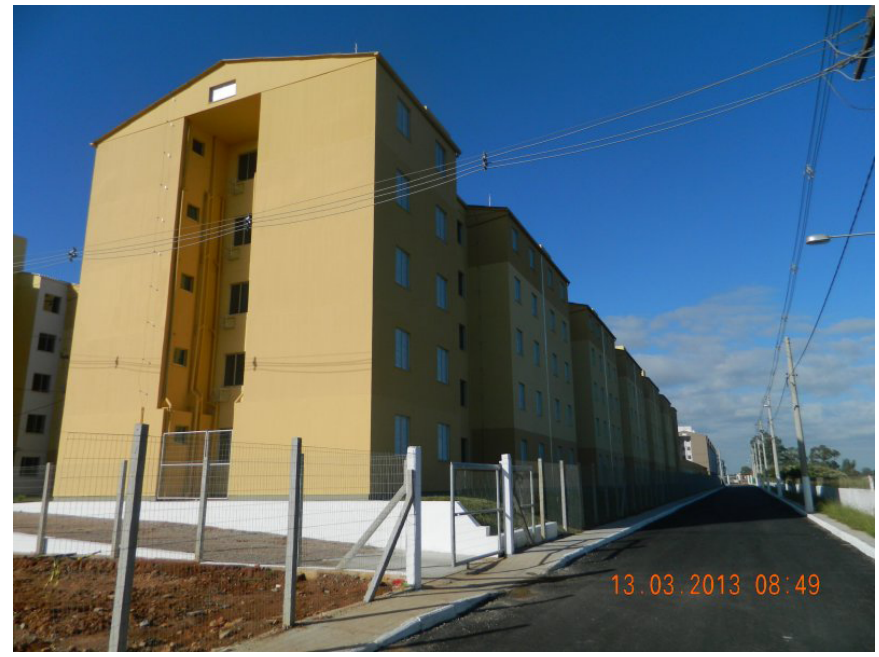

Fuente: Labore Ingeniería (2013).

Los emprendimientos realizados por la empresa Labore en el marco del PMCMV se diferencian de aquellos efectuados bajo el PAR cuantitativamente, en función de la densidad habitacional y del número de viviendas que aumentó, y cualitativamente con relación a la inserción de los proyectos en el tejido urbano por su localización periférica. En ese sentido, Chiarelli (2014), afirma que los residenciales Montevideo y Buenos Aires constituyen los proyectos más distantes en relación al centro de la ciudad, característica que implica mayores dificultades para los residentes en el acceso a los equipamientos y servicios urbanos disponibles, afectando las condiciones de inclusión y los costos sociales. Sin embargo, la localización periférica sigue las estrategias de producción de la empresa ya que potencializa la ampliación de los lucros por la utilización de la reserva propia de suelo urbano (banco de tierras) y por su bajo precio, contribuyendo al mismo tiempo con la valorización del suelo urbano remanente.

Roberto Ferreira Comercial y Constructora es la segunda compañía con mayor presencia en la modalidad Empresas del PMCMV, tanto en números de proyectos como en viviendas construidas, alcanzando 700 unidades en dos emprendimientos. Actuando desde 1953 constituye la empresa más antigua en ejercicio y con una amplia variedad de productos, que incluye viviendas de alto estándar, inmuebles de uso comercial y conjuntos de viviendas populares de gran escala. Desde su fundación esta constructora se destaca en el mercado local por su asociación con familias tradicionales de la ciudad, específicamente, grandes propietarios de tierras y de propiedades urbanas que iniciaron la conversión del uso del suelo al final de los años 1950. Según Soares (2002) esta asociación entre la constructora y los propietarios permitió la renovación urbana en el centro histórico y la expansión de los negocios inmobiliarios y del tejido urbano hacia el sector oriente de la ciudad en un área de terrenos inundables, dejando grandes vacíos urbanos intermediarios para su valorización.

En 2009 la constructora Roberto Ferreira anunció el Residencial Eldorado, primer proyecto del PMCMV Empresas finalizado en Pelotas en 2011. Este proyecto inmobiliario fue edificado en un 
vacío urbano de aproximadamente 11 hectáreas del sector oriente de la ciudad de propiedad de la Municipalidad de Pelotas. El proyecto consideraba la construcción de 420 viviendas de albañilería pareadas, con placas solares y una central de colecta y tratamiento de aguas servidas, y se destinó para familias que ocupaban un terreno público.

Aunque la materialización del residencial Eldorado consideró infraestructura urbana básica en las áreas comunes, desde su entrega en 2011 los residentes han denunciado al Ministerio Público y a los medios de comunicación una serie de problemas relacionados con la mala calidad de los equipamientos. Según el presidente de la Asociación de Vecinos, los principales problemas son la ausencia de una línea de buses en el barrio, la inseguridad, el escape de las aguas servidas, la destrucción de las calles y la ausencia de una unidad básica de salud. En las Figuras № 3 y 4 es posible observar las marcas de la violencia en un muro con marcas de tiros y la destrucción de las calles.

Figura $N^{\circ} 3$

Residencial Eldorado: casa con marcas de tiros.

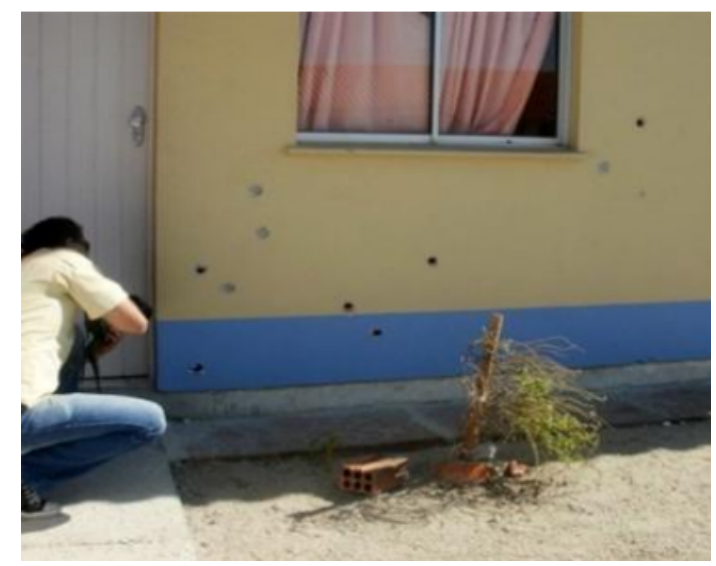

Fuente: Blog Amigos de Pelotas (2012).

Figura $\mathrm{N}^{\circ} 4$

Residencial Eldorado: calle con pavimento destruido.

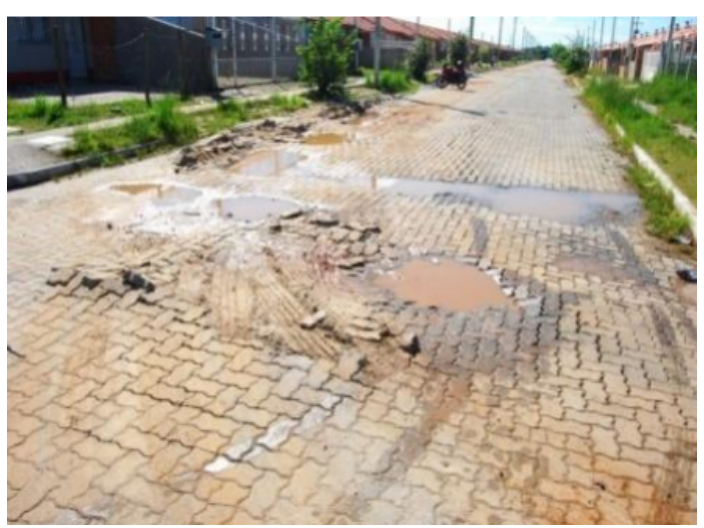

Fuente: Blog Amigos de Pelotas (2012). 
Un reportaje del Diario Popular ${ }^{11}$ en 2014 estimó que cerca de la mitad de las 420 viviendas no eran ocupadas por los beneficiarios originales en función de la ausencia de fiscalización por parte del poder público municipal y de la Caixa Económica Federal, responsable por los contratos. Por su parte destacaba que la responsabilidad sobre los problemas y su solución estaba marcada por la divergencia entre la empresa constructora y el poder público, ya que la empresa acusaba a la Municipalidad y los vecinos por la falta de mantención, mientras que el poder público municipal alegaba que los problemas se debían a fallas estructurales de la obra, especialmente en el caso del derrame de las aguas servidas.

En 2014 la constructora Roberto Ferreira entregó el Residencial Haragano (Figura № 5), su segundo proyecto del PMCMV en Pelotas, con 280 viviendas localizadas en un área de vacío urbano en la zona norte de la ciudad. El terreno donde fue construido este conjunto fue donado por el Gobierno del Estado de Rio Grande do Sul por medio del proyecto Banco de Tierras Públicas ${ }^{12}$, mientras que la licitación para elegir la empresa constructora fue realizada por el Gobierno Municipal de Pelotas, caracterizando el primer emprendimiento que significó una articulación entre los niveles federal, estadual y municipal de la administración pública. Este proyecto también demuestra una articulación con el sector inmobiliario local, ya en que la zona donde se localizó el Residencial Haragano existían otros terrenos libres pertenecientes a las empresas interesadas en la expansión de la ciudad y la valorización de sus propiedades.

Figura $\mathrm{N}^{\circ} 5$

Residencial Haragano.

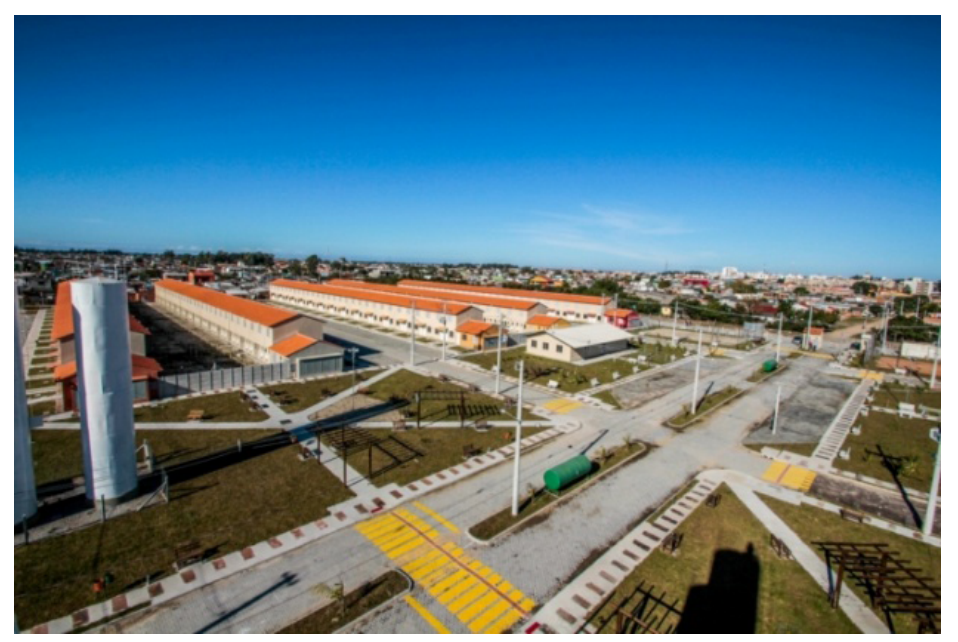

Fuente: Municipalidad de Pelotas.

En términos constructivos, el Residencial Haragano se caracteriza por el uso de técnicas industriales de construcción estandarizada y de gran escala, mediante el uso de un sistema de

\footnotetext{
Reportaje publicado el 10 de noviembre de 2014. Fuente: https://www.diariopopular.com.br/geral/para-qualificar-o-programa-foco-no-cadastro-92046/? Acceso en 25/01/2017.

12 El proyecto Banco de Tierras Públicas fue creado en 2011 con la finalidad de estimular la donación de áreas públicas de dominio del gobierno estadual para la construcción de viviendas sociales.
} 
paneles estructurales de madera y yeso (Figura $N^{\circ} 6$ ). Esa técnica ofrece reducción de costos en función del menor tiempo empleado en la construcción y el menor uso de mano de obra, viabilizando un mayor margen de lucro en la producción de viviendas sociales de bajo costo. De acuerdo con la constructora, este fue el primer proyecto del PMCMV construido totalmente con esa técnica en Brasil. Además, según Espíndola e Ino (2014), para el financiamiento de construcciones con tecnologías aún no estandarizadas en el país, fue necesario modificar las normas técnicas ${ }^{13}$ nacionales a través de la institución del Sistema Nacional de Evaluación Técnica (SINAT), siendo deliberado este cambio de acuerdo con los parámetros normativos específicos propuestos por la empresa Roberto Ferreira, evidenciando así el papel político de esta empresa local en la escena nacional.

Figura $\mathrm{N}^{\circ} 6$

Técnica constructiva del Residencial Haragano.

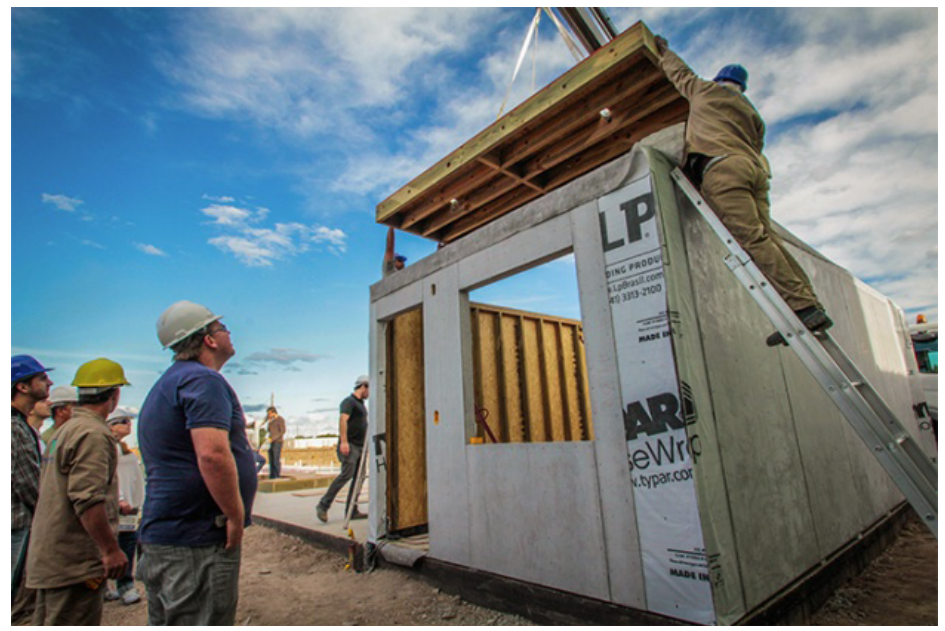

Fuente: Municipalidad de Pelotas.

La tercera empresa en volumen construido en Pelotas corresponde a Serial Ingeniería y Estructuras, con dos proyectos y 520 viviendas. La empresa fundada en 1984 actúa en diversos ramos de la construcción civil, inclusive en la actividad metalúrgica con la fábrica Sermetal Industria de Estructuras, donde son fabricadas escuadras metálicas en aluminio y acero para diferentes usos, y sistemas modulares en PVC. En las actividades de construcción y promoción inmobiliaria, Serial posee un know-how diversificado, actuando en la producción de conjuntos habitacionales de vivienda social, de condominios horizontales para grupos de ingresos medios y altos - especialmente mediante la constitución de Sociedades de Propósitos Específico ${ }^{14}$ (SPE) -, de edificios comerciales, industriales y obras públicas.

\footnotetext{
13 Entre diciembre de 2019 y febrero de 2020 (mientras este artículo aún estaba en prensa), el residencial Haragano sufrió dos incendios importantes, dejando a más de 30 familias sin hogar. Las autoridades locales aún no han revelado las causas del incendio, pero todo indica que el uso de la estructura de madera fue decisivo para propagar el incendio entre las viviendas.

14 Modelo de organización empresarial por el cual se constituye una nueva empresa con un objetivo específico, cuya actividad se limita a un único proyecto, pudiendo definir un plazo de existencia determinado.
} 
El Residencial Amazonas fue contratado en 2013 con la constructora Serial y gestionado desde el punto de vista financiero por el Banco do Brasil. Corresponde a un conjunto habitacional de 280 departamentos construidos en edificios de cinco pisos. La localización de este proyecto merece un análisis más detallado, ya que se ubica en una zona periférica de características rururbanas, vecino a otro residencial periférico (Figura $N^{\circ} 7$ ) - Residencial Roraima da la constructora Labore - que, así como los conjuntos Montevideo y Buenos Aires, constituyen las áreas residenciales más distantes del centro de la ciudad producidos en el ámbito del PMCMV en Pelotas.

Figura № 7

Residenciales Amazonas y Roraima localizados en área periférica de características rururbanas.

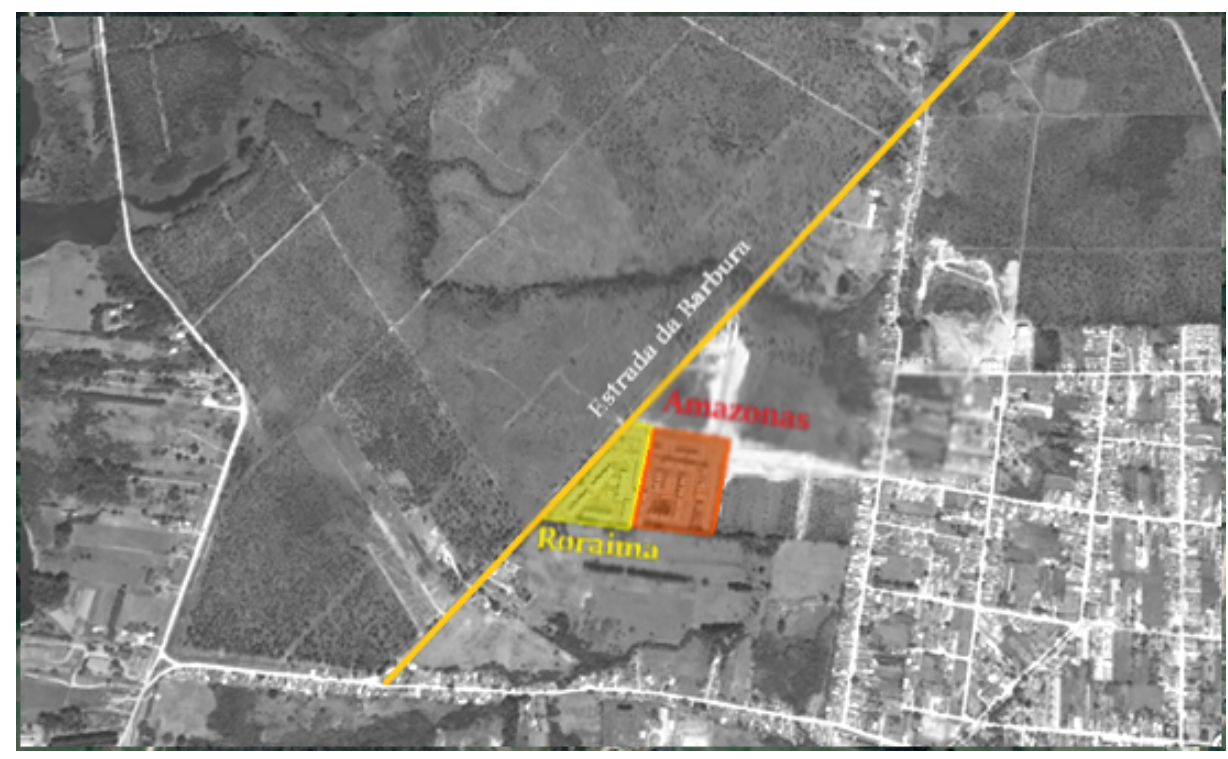

Fuente: Google Earth (2017).

El área donde se localizan estos residenciales se caracteriza por su uso predominantemente rural, con la presencia de actividades agropecuarias, además de la extracción de arena y arcilla. Legalmente correspondió a un área rural, por lo tanto con prohibición legal de ser loteada y destinada al uso residencial urbano, hasta finales del año 2012. Coincidentemente el día 28 de diciembre de 2012, faltando tres días para acabar el mandato del alcalde de la época, fue aprobada la ampliación del límite urbano incorporando las tierras pertenecientes a las empresas Labore Ingeniería y Serial Ingeniería. Seis meses después parte de esas tierras recibieron emprendimientos del PMCMV.

Si bien la empresa Serial obtuvo ganancias excepcionales con la formación de un banco de tierras mediante la conversión de condición legal del suelo - antes rural y ahora urbano -, también desarrolla estrategias constructivas que le permiten multiplicar sus lucros, ya que utiliza sistemas constructivos industrializados y de bajo costo. En efecto, sus viviendas se caracterizan por sus estructuras de plástico reforzado con concreto, denominadas en Brasil como concreto PVC, y por la utilización de placas estructurales de plástico reforzado con fibra de vidrio.

A partir de los ejemplos analizados es posible evidenciar algunas articulaciones que se establecen entre los agentes públicos y privados en la producción del PMCMV destinado a la po- 
blación de menores ingresos (grupo 1) en la ciudad de Pelotas. En primer lugar se destacan las modificaciones a la legislación urbana para encuadrar los proyectos inmobiliarios dentro de las exigencias de la política pública y la omisión de los agentes públicos en el combate a las prácticas que no respetan las normas del programa, como se puede verificar en los atrasos para la entrega de los emprendimientos, en la falta de fiscalización de la ejecución del trabajo técnico, en el no acompañamiento de las condiciones posteriores a la ocupación de las viviendas y en los problemas originados por la localización periférica y desconectada del tejido urbano consolidado. En efecto, de los diez residenciales construidos en la ciudad por la modalidad Empresas, cinco se localizan en el sector norte de la ciudad y cuatro de ellos se emplazan a más de 10 kilómetros del centro de la ciudad; otros cuatro conjuntos se concentran en el sector noreste en un área de vacío urbano contribuyendo con la valorización de las tierras sin ocupación y definiendo un vector de expansión periférico en una región carente de infraestructura (Figura No 8).

Figura $N^{\circ} 8$

Localización de los proyectos PMCMV Grupo 1.

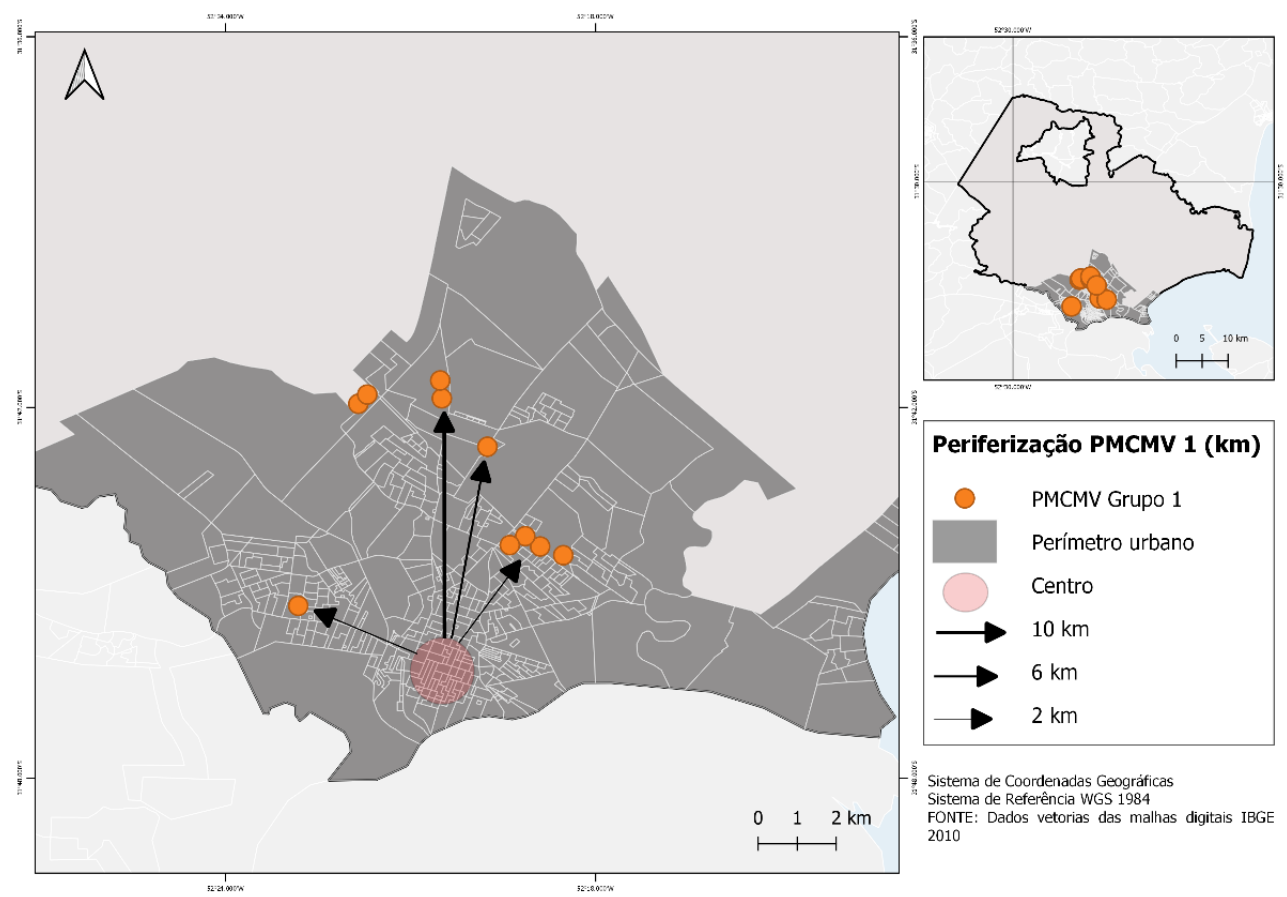

Fuente: Autores (2018).

De ese modo, en función de la escasa reglamentación y fiscalización del PMCMV en Pelotas, se constata la libre actuación de las empresas locales que pasan a direccionar sus estrategias para la transformación de la tierra rural en suelo urbano, como táctica principal para lucrar y dinamizar el proceso de acumulación de capital. En la misma dirección, las empresas pasan a racionalizar e industrializar los procesos constructivos con el uso de materiales prefabricados y técnicas organizacionales de bajo costo, que aparentan la "modernización" de un sector productivo históricamente tradicional.

En síntesis, la articulación de intereses privados y la oferta de financiamiento público - vía subsidios a la demanda -, explican el interés de las constructoras por la producción de viviendas para 
este estrato de ingresos, especialmente de empresas locales que además de conocer el mercado y participar de las redes locales de poder, disponen de bancos o reservas de tierras rurales con potencialidad de transformarse en suelo urbano. Del mismo modo en el actuar de esas empresas se sobreponen los papeles de propietarios de tierras, promotores inmobiliarios y constructores que caracteriza la constitución del sector inmobiliario de Pelotas.

\section{PMCMV Modalidad Fondo de Garantía por Tiempo de Servicio (Grupos 2 e 3)}

A diferencia de las viviendas para el grupo de menor poder adquisitivo (grupo 1), en que prevalecían empresas de carácter local, en las modalidades del PMCMV destinadas a los grupos de ingresos entre 3 y 10 sueldos mínimos (grupos 2 y 3) se observa la actuación de empresas regionales y nacionales. Esta característica impactó las empresas locales que debieron ajustar sus procesos organizacionales para participar con un volumen mayor de producción y en escala ampliada, considerando el importante nicho de mercado que se creó para la demanda en este segmento de ingresos, sea para la demanda que se autofinancia o para la demanda que vislumbró la compra de una vivienda a partir de las líneas de crédito facilitadas por el PMCMV.

El total de unidades habitacionales contratadas hasta 2016 en Pelotas, destinadas para los grupos 2 y 3, llegó a 8.103 viviendas, equivalentes a 71\% de todas las unidades contratadas en la ciudad, consumiendo casi $80 \%$ de los recursos del PMCMV. En el grupo 2, que se destina a familias con ingresos entre 3 y 6 sueldos mínimos, fueron construidos 33 emprendimientos, con 6.866 viviendas. Para el grupo 3, de familias con ingresos entre 6 y 10 sueldos mínimos, se construyeron siete emprendimientos con un total de 1.237 viviendas (MCidades, 2016).

Con la implementación del PMCMV el mercado inmobiliario de Pelotas, que se caracterizó históricamente por un cierto grado de aislamiento, pasó a vivenciar la entrada de nuevos agentes y nuevas estrategias de producción que influyeron de forma articulada en la valorización inmobiliaria local. Entre esos nuevos agentes se destaca la empresa Rodobens Negocios Inmobiliarios, una de las seis mayores constructoras de Brasil, que actúa en la ciudad desde 2009 produciendo condóminos horizontales para la denominada "nueva clase media-baja brasileña" o clase C.

Con sede en la ciudad paulista de São José do Rio Preto, la promotora y constructora forma parte del conglomerado Rodobens S.A., un holding compuesto por ocho empresas que actúan en diversos sectores, desde el arriendo y venta de vehículos, corredoras de seguros, consorcios, trading company, hasta un banco propio. En 2007 la empresa realizó su oferta pública inicial de acciones, levantando cerca de $\mathrm{R} \$ 450$ millones, especialmente entre inversionistas extranjeros que pasaron a controlar $75 \%$ de sus acciones. Esta capitalización le permitió elevar su capacidad productiva y de expansión en el mercado, pasando a actuar fuertemente en el segmento de producción de viviendas económicas en ciudades con más de 100.000 habitantes. Así Rodobens Negocios Inmobiliarios alcanzó, hasta 2016, una producción de 170 emprendimientos residenciales, con más de 64.000 unidades habitacionales, distribuidas en más de 50 ciudades de 12 estados brasileños (Rodobens, 2016).

En Pelotas, hasta 2016, Rodobens fue responsable por la contratación de 3.754 viviendas en ocho proyectos residenciales: Terra Nova, Moradas, Moradas 2, Moradas Club, Moradas Club 2 y 
Moradas Club o Ildefonso II, III y IV. Ese volumen coloca esta empresa como la mayor constructora en número de viviendas contratadas en la ciudad.

El primer emprendimiento lanzado fue Terra Nova, un condominio de casas unifamiliares pareadas destinado a atender familias con ingresos a partir de cinco sueldos mínimos, con financiamiento inmobiliario administrado por Rodobens S.A. en asociación con el Banco del Estado de Rio Grande do Sul (Banrisul) y más tarde transferido para el PMCMV. Los restantes residenciales Rodobens se orientaron para un público con ingresos a partir de tres sueldos mínimos, adecuándose a los grupos de ingresos definidos por el programa habitacional.

En términos constructivos la empresa Rodobens se caracteriza por utilizar un método estandarizado posibilitando mayor rapidez en la producción de las viviendas. En 2012 el proceso incorporó la utilización de siding vinílico o revestimiento en PVC consiguiendo aumentar la velocidad de la construcción, estimada en dos casas por día, permitiendo la obtención de mayor lucro.

Los tres primeros lanzamientos de Rodobens - condominios Terra Nova, Moradas y Moradas Club - significaron 1.666 unidades habitacionales construidas en una misma gleba de aproximadamente 30 hectáreas (Figura $N^{\circ} 9$ ), localizada en un vacío urbano entre el centro de la ciudad y los barrios periféricos consolidados. La inserción de estos proyectos en la trama urbana trajo problemas de movilidad y un fuerte aumento en la densidad demográfica.

Figura $\mathrm{N}^{\circ} 9$

Localización de los condominios Terra Nova, Moradas y Moradas Club

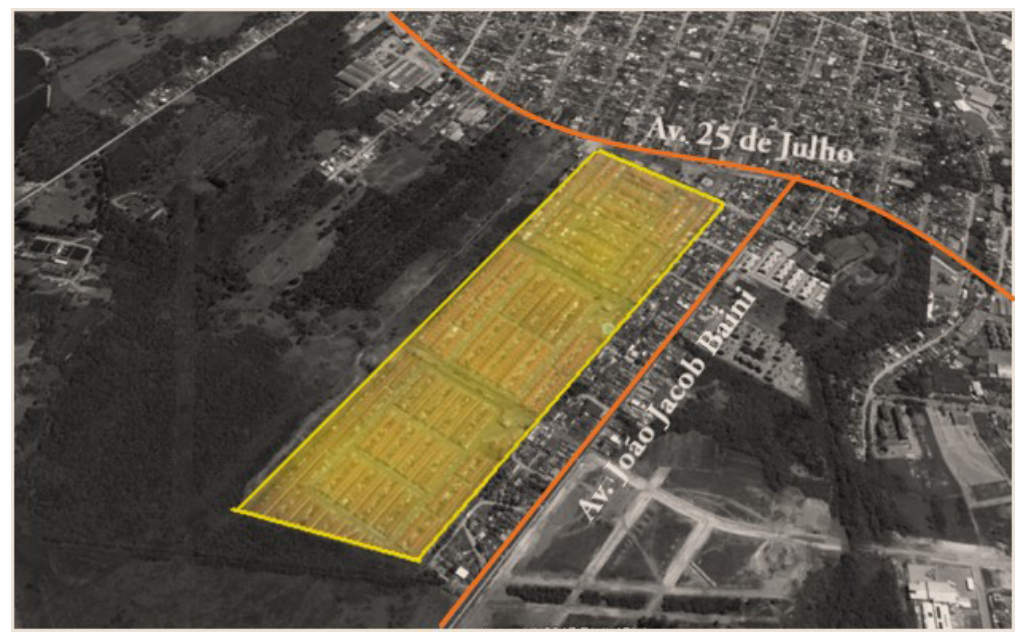

Fuente: Google Earth (2016).

El estudio realizado por Berger et al (2014) demuestra algunos de los impactos en el entorno provocados por estos condominios. Entre los problemas identificados se destacan la insuficiencia de transporte colectivo y del sistema vial y la deficiente oferta de equipamientos y servicios urbanos instalados en las proximidades, como áreas verdes, escuelas y unidades básicas de salud. Al interior de estos proyectos residenciales, los condominios han sido usados para burlar la ley de división del suelo que define mayores exigencias para las áreas de uso común, circulación, imple- 
mentación de equipamientos urbanos y comunitarios y áreas verdes, especialmente en proyectos que aumentan considerablemente la densidad habitacional.

El gran vacío urbano que recibió los proyectos de Rodobens, entre otros proyectos residenciales de gran tamaño, se mantuvo como reserva de tierra ociosa a lo largo del proceso de estructuración y expansión de la ciudad, formando una gran gleba descontinua y no inserida en la trama urbana, aun cuando se localizaba al interior del límite urbano. Para incorporar estos proyectos residenciales fue necesaria una serie de modificaciones a la legislación municipal como forma de permitir su adecuación a las directrices y exigencias del PMCMV.

Entre esas modificaciones se encuentra la flexibilización Plan Director de Pelotas, que en su versión original limitaba en 1,5 hectáreas la superficie máxima de los condominios horizontales, con vistas a cohibir los excesos relacionados con la densidad, la expansión de la trama urbana y la segregación. En 2009 fue aumentada la superficie máxima hasta 5 hectáreas, sin obligación de una reserva de área para uso público, y en 2012 se posibilitó la ampliación de la superficie máxima para proyectos de condominios horizontales en hasta 35 veces, incluyendo proyectos de vivienda social y proyectos de alto padrón de iniciativa y financiamiento privado.

Después de esas modificaciones, la empresa Rodobens desarrolló otros cinco proyectos residenciales en otra área de vacío urbano. La misma área recibió más de 3.200 nuevas viviendas de programas sociales con los emprendimientos de Rodobens y otras empresas como Roberto Ferreira, Zecon y Serial; además concentró viviendas de proyectos privados constituyendo un sector de valorización inmobiliaria.

En síntesis, la actuación de la empresa Rodobens se destaca por la introducción del modelo de condominio horizontal en gran escala para el segmento de bajos y medios ingresos, estimulando la conformación de un nuevo hábitat urbano, caracterizado por "nuevas formas de vivir e aprehender la ciudad" (Sposito, 2013, p. 44), y nuevas lógicas de producción del espacio urbano asentadas en la ampliación de la división del trabajo (separación entre incorporación y construcción), el aumento de la racionalidad técnico-productiva (industrialización del proceso productivo) y el estrechamiento de la relación entre los procesos de financiarización de las empresas, de la propiedad y de la vivienda.

Para eso la actuación de la empresa estuvo condicionada a su articulación con agentes públicos y privados locales y regionales a través de redes de cooperación, joint-ventures e sociedades de propósitos específicos, con los objetivos de: acceder a los terrenos - land banks - controlados por propietarios de tierras y empresas locales, adecuar y aprobar los proyectos en las instancias municipales y facilitar su entrada al mercado local para la explotación de un nicho de consumo habitacional específico. En ese sentido, fue fundamental la articulación de Rodobens con propietarios de tierras y empresas inmobiliarias de Pelotas, responsables por garantizar la disponibilidad de los terrenos, por los licenciamientos de los proyectos, por presionar al poder público municipal para flexibilizar las normas y por la comercialización y administración de los emprendimientos.

Como señala Amorim (2015), un obstáculo para la entrada de las grandes empresas en los mercados locales es la tierra, altamente concentrada en las ciudades pequeñas y medias; situación que se verifica en Pelotas. Sobre ese mismo tema, Rufino (2016) destaca que el acceso a los 
bancos de tierras en diferentes regiones de Brasil es una estrategia esencial para las empresas de capital abierto, ya que el control sobre la tierra ejerce influencias en la percepción de los analistas e inversionistas que relacionan el valor de las acciones de una empresa a las ganancias potenciales, proyectadas a partir de las características y del volumen de terrenos disponibles. Por su parte, Rocha Lima (2007) analiza la importancia de la constitución de land banks por medio de asociaciones con los propietarios de tierras para no inmovilizar el capital de giro de las empresas. En esas asociaciones, que pueden significar permuta por unidades habitacionales o permuta por una parte de las ventas, se produce una reducción de los riesgos que significaría la inmovilización de los recursos, permitiendo una mayor fluidez en los negocios.

En ese sentido, el acceso y control sobre la propiedad de la tierra posibilitados a Rodobens por su asociación con agentes locales, estimuló el proceso de financiarización de la empresa, al mismo tiempo transformó las lógicas de producción del espacio urbano y de la vivienda en Pelotas, estimulando una fuerte ampliación de la periferia. De igual manera, la nueva lógica productiva significa una creciente separación entre las actividades de construcción, comercialización y promoción, posibilitando una mayor expansión geográfica y productiva de las empresas. Por su vez, la asociación con los grandes grupos de actuación nacional significa para los agentes locales y regionales la posibilidad de acceder a lucros excepcionales vinculados a la construcción estandarizada, a la valorización del suelo urbano, a la comercialización asociada a campañas de marketing y a la administración de los condominios.

Con relación a los lucros excepcionales por concepto de comercialización es importante señalar que, diferentemente de lo que acontece en el grupo 1 del PMCMV, en el cual no existe la comercialización, ya que la municipalidad selecciona los beneficiarios, en los grupos 2 y 3 el agente promotor debe vender las viviendas y su retorno financiero depende de esa comercialización. En ese sentido, la actuación coordinada con los agentes vendedores locales es fundamental, debido al control que estos ejercen sobre las carteras y stocks de inmuebles, además de su conocimiento y contactos en el mercado local.

Para las grandes constructoras surge otro frente de lucros, asociado a las ganancias financieras de las empresas de capital abierto que valorizan sus acciones a través de las proyecciones de productividad, del número de proyectos en ejecución y de la distribución geográfica de los mismos, ya que el mercado valoriza la escala de producción y la escala geográfica de actuación de las empresas.

Finalmente, en términos de los impactos en la dinámica de los precios y la valorización de los terrenos urbanos en la ciudad, como resultado principalmente del aumento en la producción de viviendas del PMCMV, se destaca el aumento porcentual en los precios de las propiedades en el período analizado, especialmente en los sectores periféricos que recibieron los proyectos del programa. Entre 2009 y 2016, el sector norte, que recibió 50\% de las viviendas del grupo 1 y $34 \%$ de los grupos 2 y 3 , tuvo una apreciación de 198\% en el período, siendo el segundo sector de mayor valorización inmobiliaria en la ciudad. El sector noreste, el segundo en concentración de lanzamientos en el programa, tuvo la tercera mayor apreciación en el período, con valores cercanos a los observados en el área central de la ciudad. Finalmente, vale la pena mencionar que el sector oriental, que tuvo la mayor valorización en el período, no obtuvo ningún lanzamiento del PMCMV ya que se caracteriza como el sector de altos ingresos, con un predominio de lanzamientos de 
bienes raíces de alto estándar, especialmente del tipo de condominios horizontales y barrios cerrados (Figura No 10).

\section{Figura No 10}

Localización del PMCMV y porcentaje de valoración de suelo urbano ${ }^{15}$ entre 2009 y 2016.

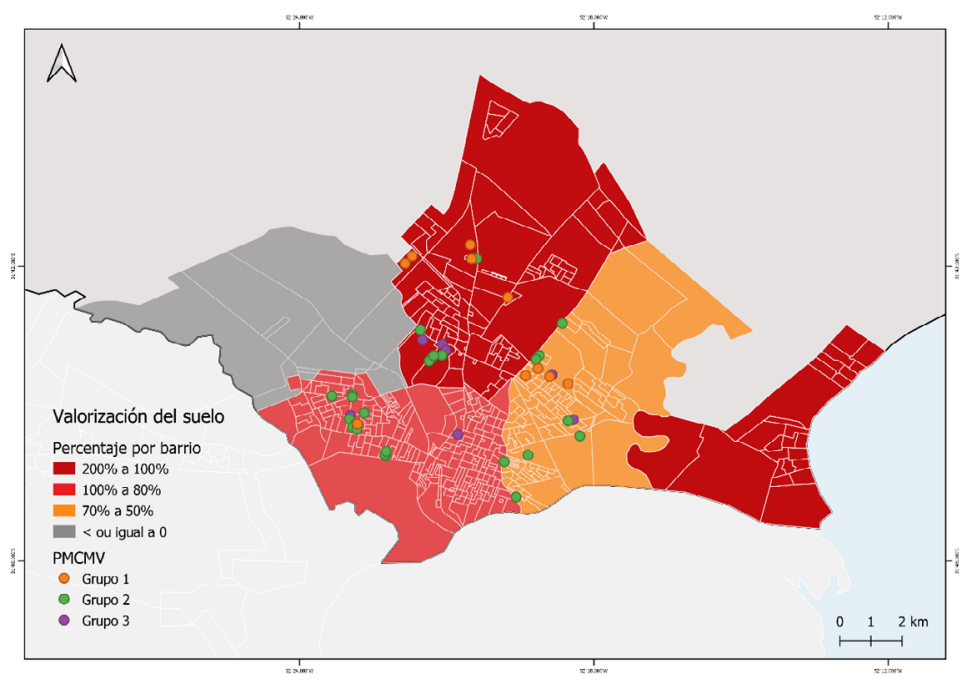

Fuente: Autores (2018).

\section{Consideraciones finales}

La expansión del sector inmobiliario verificada en los últimos años se realiza cada vez más relacionada con la actuación del Estado - por sus políticas habitacionales - que posibilita una dinámica más intensa en la producción del espacio en las ciudades brasileñas. En Pelotas, las empresas del ramo de la construcción orientan su producción de modo diversificado, atendiendo las demandas de diferentes segmentos de ingresos, lo que da como resultado una tipología habitacional diversa en el paisaje urbano, así como la proliferación de nuevos procesos socio-espaciales que se destacan en la estructura de la ciudad.

Como fue analizado, además de la solvencia de la demanda, existen factores y especificidades del sector inmobiliario de Pelotas que estimulan la producción para los grupos 2 y 3 del PMCMV, en detrimento del grupo 1 donde se concentra el déficit habitacional de la ciudad. Entre los factores generales está el gran volumen de recursos destinados a los segmentos de más de tres sueldos mínimos, situación que se relaciona directamente con la ampliación, desde 2003, de los ingresos fiscales destinados a los programas habitacionales en función de la coyuntura macroeconómica favorable y de ascensión de la llamada nueva clase trabajadora (Pochmann, 2012). Otra condición favorable a la expansión inmobiliaria fue la propia creación en 2009 del PMCMV,

El porcentaje de valorización del suelo urbano se basó en datos de ITBI, agregados y corregidos por el índice IGP-M (FGV) para el período de análisis. El IGP-M (índice general de precios de mercado) es una de las versiones del Índice General de Precios (IGP). Lo mide la Fundación Getulio Vargas (FGV) y registra la inflación de los precios desde las materias primas agrícolas e industriales hasta los bienes y servicios finales. 
que permitió la aceleración de la rotación de capitales en el sector, antes inmovilizados por los sistemas de financiamiento directos al consumidor. Con el programa habitacional, que garantizó subsidios públicos y retorno financiero, se amplió el número de emprendimientos asumidos por las constructoras.

En el caso específico de Pelotas, las estrategias de los agentes participantes en la producción habitacional del PMCMV significan condiciones ampliadas de acumulación que se realizan en los diferentes frentes y que movilizan una diversidad de agentes con diferentes intereses, tamaños y escalas de actuación, pero que participan de forma articulada en la producción y distribución de los lucros provenientes de la promoción inmobiliaria, del control del mercado de tierras, de la construcción y de la valorización financiera.

En función de las características estructurales de la ciudad se destaca también el carácter central de la tierra en el proceso de incorporación de los proyectos residenciales, tanto en la ampliación de la valorización inmobiliaria, como en la valorización financiera a través de la cotización de las empresas con base en su patrimonio y en las proyecciones del control que ejercen sobre el suelo urbano. En ese sentido, el PMCMV potencializa procesos de producción inmobiliaria, habitacional y de la ciudad a partir de las ganancias obtenidas en el plano financiero, situación que explica, hasta cierto punto, el interés de grandes grupos en los mercados locales y regionales, fuera de sus áreas de origen, así como la característica oligopólica del sector.

Para finalizar, es importante enfatizar el éxito cuantitativo alcanzado por el PMCMV en Pelotas, especialmente en los segmentos de ingresos medios, permitiendo el acceso a la vivienda a un grupo expresivo de la población que hasta ese momento solamente podía acceder al mercado inmobiliario privado sin subsidios o financiamientos especiales. De igual manera, es necesario relativizar las críticas al programa considerando que una política de impacto nacional, en un país de tamaño continental como Brasil, difícilmente sería materializada sin suscitar una serie de contradicciones inherentes al proceso de producción de la vivienda y propias de las relaciones de poder, como se puede observar en los trabajos de Marques y Rodrigues (2013) y Jesus y Denaldi (2018). Sin embargo, también es necesaria la reflexión sobre los límites de una política que se fundamenta en el modelo de propiedad privada y de estímulo a la creciente financiarización de la producción habitacional. Como señalan autores como Shimbo (2010, 2016), Rolnik (2015) y Santos (2015), ese modelo tiende indiscutiblemente a reducir el papel de la vivienda a propiedad y a lastro para operaciones de crédito, ya que en su esencia engendra la lógica de mercado y de la acumulación expansiva, atendiendo de forma prioritaria a los intereses económicos sectoriales y de las finanzas. De esa forma, no es de extrañar que la forma predominante de producción habitacional en Brasil se mantenga distante del combate efectivo al déficit habitacional y que se aleje cada vez más del ideal del derecho a la ciudad, que implica además de las condiciones del hábitat, el derecho a habitar en un sentido amplio, considerando la vivienda digna, la movilidad urbana, la inserción en la ciudad existente y la sociabilidad urbana.

\section{Referencias bibliográficas}

AMORIM, W. A produção imobiliária e a reestruturação das cidades médias: Londrina e Maringá/ $P R$. Tesis doctorado en Geografía. Presidente Prudente: UNESP, 2015. 
ARAÚJO, T. B. Desenvolvimento regional brasileiro e políticas públicas federais. En: Sader, E. (org). 10 anos de governos pós-neoliberais no Brasil: Lula e Dilma. São Paulo: Boitempo, 2013, p. 157-171.

BASTOS, P. A economia política do novo-desenvolvimentismo e do social desenvolvimentismo. Economia e Sociedade, № 21, 2012, p. 779-810.

BERGER, J. ET AL. Avaliação da gestão pós-ocupação estudo de caso: condomínios do Programa Minha Casa Minha Vida em Pelotas-RS. Maceió: Anais XV ENTAC, 2014.

BRESSER-PEREIRA, L. C. Do antigo ao Novo Desenvolvimentismo na América Latina. São Paulo: FGV-EESP, 2010.

BRASIL. Cartilha Minha Casa, Minha Vida. Brasília: Ministério das Cidades, 2014.

BRASIL. Empreendimentos e valores do Programa Minha Casa Minha vida, faixa 1, 2 e 3. Brasília: MCidades, 2016.

CARDOSO, A. \& ARAGÃO, T. Reestruturação do setor imobiliário e o papel do Programa Minha Casa Minha Vida. Salvador: Anais XIII Seminário Internacional RII, 2014.

CHIARELLI, L. M. A. Habitação social em Pelotas (1987-2010): influências das políticas públicas na promoção de conjuntos habitacionais. Tesis doctorado en Historia. Porto Alegre: PUCRS, 2014.

ESPINOLA, L \& INO, A. Inserção e financiamento do sistema Wood frame do programa habitacional Minha casa minha vida. Porto Alegre: Associação Nacional de Tecnologia do Ambiente Construído - ANTAC, 2014. Disponible en: http://www.infohab.org.br/entac2014/artigos/paper_566.pdf

GONÇALVES, R. Novo desenvolvimentismo e liberalismo enraizado. Serviço Social \& Sociedade, 2012, NN 112, p. 637-671

JESUS, P. \& DENALDI, R. Experiências de regulação urbana e suas possibilidades: análise a partir do Programa Minha Casa Minha Vida na Região do Grande ABC (São Paulo). EURE, 2018, № 44(132), p. 91-111.

ROCHA LIMA, JR. Landbank das empresas de Real Estate listadas na Bovespa. Escola Politécnica da Universidade de São Paulo. 2007. Disponible en: https://www.realestate.br/dash/uploads/sistema/ images/File/arquivosPDF/Pini_colunadeNovembro07_Joao.pdf

MARQUES, E. \& RODRIGUES, L. O Programa Minha Casa Minha Vida na metrópole paulistana: atendimento habitacional e padrões de segregação. Revista Brasileira de Estudos Urbanos e Regionais, 2013, № 15(2), p. 159-177. https://doi.org/10.22296/2317-1529.2013v15n2p159

PINASSI, M. O. Nas sombras do obscurantismo. Margem Esquerda, 2013, № 1, p. 91-101.

POCHMANN, M. Nova Classe média? O trabalho na base da pirâmide social brasileira. São Paulo: Boitempo, 2012. 
RODOBENS. ITR - Informações Trimestrais. Releases Rodobens NI National Rating. 2016. Disponible en: http://ri.rni.com.br/conteudo_pt.asp?idioma=0\&conta=28\&tipo=19616\&id=0\&submenu=0\&img=0\&ano $=2016$

ROLNIK, R. A Guerra dos lugares: a colonização da terra e da moradia na era das finanças. São Paulo: Boitempo, 2015.

RUFINO, B. Incorporação da metrópole: transformações na produção imobiliária e do espaço na Fortaleza do século XXI. São Paulo: Annablume, 2016.

SANTOS, C. R. A fronteira urbana: urbanização, industrialização e mercado imobiliário no Brasil. São Paulo: Annablume, 2015.

SHIMBO, L. Z. Habitação Social, Habitação de Mercado: a confluência entre Estado, empresas construtoras e capital financeiro. Tesis Doctorado en Arquitectura y Urbanismo. São Carlos: USP, 2010 .

SHIMBO, L. Z. Sobre os capitais que produzem habitação no Brasil. Novos Estudos CEBRAP, 2016, №35(2), 119-133. http://dx.doi.org/10.25091/s0101-3300201600020007

SOARES, P. Del proyecto urbano a la producción del espacio: morfología urbana de la ciudad de Pelotas, Brasil (1812-2000). Tesis doctorado en Geografía. Barcelona: Universidad de Barcelona, 2002.

TRENTO, M. \& LEITÃO, K. Precariedade habitacional: uma leitura a partir de três cidades médias no sul do Brasil. São Paulo: FAUUSP, 2013. 
